\title{
CUESTIONARIO SOBRE CLIMA ORGANIZACIONAL UNIVERSITARIO.
} CUALIDADES PSICOMETRICAS

QUESTIONNAIRE ON UNIVERSITY ORGANIZATIONAL CLIMATE. PSYCHOMETRIC QUALITIES

Marialida Mujica de González ${ }^{1}$

UCLA

Isabel Pérez de Maldonado**

UPEL-IPB

Núcleo de Investigación en Comportamiento Organizacional

Recibido: 05-11-07

\section{RESUMEN}

Con el propósito de proveer a gerentes de las universidades con una medida del clim organizacional, válida, confiable y oportuna para la toma de decisiones, este estudio analiza las propiedades psicométricas de un cuestionario de clima organizacional universitario $\left(\mathrm{C}_{\mathrm{CLIOU}}\right)$. Éste fue respondido por 271 profesores de la Universidad Centroccidental "Lisandro Alvarado". El C $\mathrm{C}_{\mathrm{CLOU}}$ en su primera versión estuvo conformado por 118 ítemes. El análisis del $\mathrm{C}_{\mathrm{C} \text { ou }}$ reportó: (a) validez de itemes. El anásís del CLIou reportó. (a) validez de contenido según juicio de expertos (12 profesionales lo validaron) $y$ coeficiente de Kendall ( $\mathrm{W}=0,90$ ) (b) poder discriminativo en los ítemes; (c) una estructura bifactorial para cada uno de los componentes del clima: Imagen Gerencial, Relaciones Sociales, Toma de Decisiones y Tarea; y (d) confiabilidad de 0,97 . En el proceso de análisis se excluyeron 54 ítemes. Las propiedades psicométrica corresponden a una versión de 64 ítemes. Se concluye que el cuestionario es adecuado para medir el clima organizacional universitario.

Descriptores: Clima organizacional, diagnóstico organizacional, acción gerenci

${ }^{1}$ Profesora Titular, del Decanato de Medicina UCLA-Barquisimeto. Doctora en Educación. Magíster en Salu Pública y Magíster en Educación mención Investigación Educativa. Acreditada por el Programa de Promoción a Investigador-FONACIT Nivel 1. Miembro del Núcleo de Investigación en Comportamiento Organizaciona UPEL-IPB y Unidad de Salud Pública (Decanato de Medicina ).

** Profesora de la Universidad Pedagógica Experimental Libertador - Instituto Pedagógico Barquisimeto. Doctora en Ciencias de la Educación. Coach de Aprendizaje. Acreditada por el Programa de Promoción Investigador-FONACIT, Nivel 2. Premio a la Labor Investigación en Comportamiento Organizacional.

\section{INTRODUCCIÓN}

El clima organizacional en las universidades se caracteriza por que en el mismo se involucran diferentes elementos estructurales, funcionales y personales de la organización que, integrados en un proceso dinámico, le confieren un estilo peculiar a la gestión de la gerencia en la institución. Al estudiar el clima organizacional se recomienda que se tomen en cuenta las prácticas internas y externas de la institución ya que, como lo señala González (2000) cada una de las universidades posee características distintas; así como un conjunto complejo de factores, valores y actitudes que afectan las relaciones de las personas.

Actualmente, el clima organizacional se ha convertido en una de las áreas de estudio de mayor preeminencia, tanto en el ámbito internacional como nacional, constituyéndose en una alternativa que proporciona información multidimensional para comprender el comportamiento de las personas dentro de la organización. Uno de los aspectos más importante del clima es su visión global, pues su contenido refleja las características estructurales y funcionales de la organización, entre esas características se mencionan el tipo y el estilo de liderazgo que promueve y ejerce cualquier miembro de la organización.

En este artículo se analizan las propiedades psicométricas de un instrumento diseñado para medir el clima organizacional en la universidad. Se inicia con la discusión de algunas posiciones teóricas sobre las conceptualizaciones del clima y su medida. Posteriormente se describen detalladamente las cualidades psicométricas de un cuestionario de clima organizacional universitario $\left(\mathrm{C}_{\mathrm{CLIOU}}\right)$, obtenidas a través de procedimientos estadísticos. Se finaliza con la discusión de las evidencias de validez y confiabilidad del cuestionario y la utilidad que este brinda a la gerencia universitaria, al proveerle de información para la toma de decisiones.

\section{ALGUNAS CONCEPTUALIZACIONES SOBRE EL CLIMA ORGANIZACIONAL Y SU MEDIDA}

Los estudios de clima organizacional en el ámbito universitario han permitido identificar, categorizar y analizar las percepciones que los integrantes de una organización 
tienen de las características propias de la institución. El clima definido a través de las percepciones, está conformado por diversas dimensiones que informan tanto de la organización como de la acción gerencial. Álvarez (1992), Hall (1996), Toro (1998) y Martín Bris (2000) coinciden que es la percepción directa o indirecta que tienen los miembros de la organización. Su medición permitirá a los gerentes establecer estrategias que permitan optimizar el comportamiento organizacional.

Es importante mencionar que el clima se ha denominado de diferentes formas tales como: ambiente, atmósfera, clima universitario, clima de trabajo y clima laboral o de trabajo; sin embargo, la literatura lo reporta con mayor frecuencia con la denominación clima organizacional. González (ob.cit.) define el clima universitario como un componente multidimensional de elementos o características que identifican el sistema organizacional universitario donde interviene la conducta de las personas (gerentes, docentes, administrativos, obreros y alumnos), lo que significa que al momento de un análisis se ubica la institución como un todo, asimismo se visualiza cómo la personalidad de la institución influye en el comportamiento de las personas.

De esta manera, el clima organizacional universitario juega un papel importante en el área de desarrollo organizacional y administrativo, por cuanto su estudio permite conocer el comportamiento individual y grupal del personal en la organización y a partir de sus resultados, se diseñan mejoras con el propósito de construir un mejor ambiente de trabajo y lograr una mayor productividad, eficiencia y eficacia en la organización; por lo cual se hace necesario que en el acontecer educativo, los gerentes lleven a la práctica la comunicación, el liderazgo, la cooperación, la integración organizacional y la toma de decisiones. Todo esto se constituye en demandas perentorias para la acción gerencial y la organización universitaria.

La medición del clima organizacional suele hacerse mediante instrumentos aplicados a los trabajadores de una organización; aunque existen distintos modelos (cuestionarios, encuestas), casi todos coinciden en la medición de las percepciones que tienen del ambiente laboral. Autores entre los que se menciona, Toro (2000; 2001), exponen que existe un amplio enfoque de variables para estudiar el clima organizacional por dimensiones, del cual se derivan categorías tales como: relaciones sociales, relaciones de autoridad, tarea, claridad, retribución, retos, riesgos y participación.

Lo anterior corresponde a la literatura expuesta por Brunet (1999) quien refiere la importancia de asegurar que el instrumento empleado para la medición del clima organizacional debe incluir términos que tengan una connotación similar. Es decir, además de dimensiones, se pueden utilizar: factores, componentes, categorías e indicadores, que orienten el mayor número de preguntas al momento de elaborar el instrumento.

Los fundamentos teóricos presentados en esta investigación describen el clima organizacional como un medio para generar información útil y oportuna sobre el ambiente de trabajo en la universidad. No obstante, a pesar de la abundante literatura especializada que estudia el tema clima organizacional, las publicaciones sobre diseño y validación de instrumentos que miden este constructo son escasas; aquellos publicados se han caracterizado por definir una estructura multidimensional que refleje las características internas y externas de la organización. Una manera de medirlas es a través de cuestionarios que incluyen dimensiones del clima organizacional.

Al respecto, investigadores entre los que se mencionan Brown y Holmes (1986); Meliá e Islas (1990); Toro (1992); Coyle y otros (1995); Meliá y Sesé (1999); García (2002) han aplicado procedimientos de evaluación de los instrumentos: validez de contenido, validez de construcción y confiabilidad en organizaciones tanto empresariales como educativas, con la finalidad de obtener instrumentos seguros y estables en la medición del clima organizacional, cuyos resultados servirán de referencia en este estudio

Asimismo, Mujica de G y Pérez de M (2006) en el área de la salud, determinaron las cualidades psicométricas de un cuestionario sobre clima organizacional en los establecimientos salud ambulatoria. Reportaron que el instrumento tiene validez de contenido según el criterio de juicio de expertos y el coeficiente de concordancia de Kendall ( $\mathrm{W}=0.855$ ); los ítemes poseen poder discriminativo. También se evidenció que el instrumento conserva una estructura bifactorial, evidenciada mediante el análisis factorial confirmatorio; siendo los factores Imagen Gerencial, y la Integración Organizacional. La confiabilidad fue de 0.92 . Las cualidades reportadas llevaron a las autoras a confirmar que el cuestionario es adecuado para medir en los establecimientos de salud, las percepciones que tiene el personal sobre el clima de trabajo.

En el ámbito de la Educación Superior, Silva (2005) diseñó un instrumento para caracterizar el clima organizacional en las unidades académicas de la universidad de Colima. Utilizó como métodos de análisis del instrumento, la validación de ítemes, el coeficiente de correlación y el análisis factorial. Como resultado del proceso de estudio reportó, un instrumento estructurado en siete factores con cincuenta y nueve (59) reactivos, 
de un total de sesenta y cuatro (64) presentados inicialmente. Concluyó que el instrumento es válido y confiable para medir el clima organizacional en la universidad.

Mejias y otros (2006) desarrollaron un estudio para medir el clima organizacional en la institución de educación superior como base para introducir un modelo de sistema de gestión de calidad. Para la validez del instrumento se seleccionó el análisis de factores como método estadístico multivariado; el clima organizacional se dimensionó en tres factores: la gestión institucional, retos personales e interacción. El índice de confiabilidad fue de 0.90. Se determinó que la escala es fiable para evaluar el clima percibido por el personal de las instituciones bajo estudio.

Los estudios antes referidos aportan información útil para diseñar cuestionarios sobre clima organizacional que permita medir las percepciones que tienen los trabajadores sobre el ambiente de trabajo, lo que evidencia que no existe un modelo único, en cuanto a contenido, para diseñar cuestionarios aplicables a estudios de esta naturaleza, ni tampoco su abordaje al momento de evaluar las características, cualidades o atributos psicométricos.

En este sentido, lo planteado alcanza especial interés en las instituciones de educación superior, en particular en la universidad, porque constituye un contexto poco explorado en el campo de la investigación organizacional. Actualmente en la universidad persiste el modelo de una organización piramidal cuya relación de mando es vertical, la cual genera una serie de problemas que afectan el ambiente de trabajo y por efecto, el rendimiento, la satisfacción y la productividad del profesor.

En consecuencia, es relevante estudios de este tipo, cuyo propósito es medir de manera objetiva, la percepción que tiene el trabajador sobre el ambiente de trabajo en la organización universitaria. En el presente estudio la variable clima organizacional fue definida según el enfoque de diversos autores (Toro 1998; Gonçalves 2000; Brunet ob.cit.; Reichers y Schneider citado por Toro 2001), como el conjunto de percepciones compartidas que tienen los profesores sobre las prácticas y procedimientos organizacionales; y como el conjunto de percepciones compartidas de las condiciones y medio ambiente trabajo en la organización universitaria. Dicha variable clima organizacional, se midió mediante cuatro (4) componentes: Imagen Gerencial, Relaciones Sociales, Toma de Decisiones y la Tarea y un conjunto de subcomponentes que proporcionaron información del clima organizacional en la universidad.
Según las consideraciones expuestas, en este artículo se analizan las propiedades psicométricas de un cuestionario para medir el Clima Organizacional Universitario $\left(\mathrm{C}_{\mathrm{CLIOU}}\right)$, diseñado para tal fin, con el propósito de ofrecer al gerente una medida del clima organizacional en la universidad, válida y confiable que brinde resultados oportunos para la toma de decisiones en los procedimientos organizacionales.

\section{METODOLOGIA}

El diseño del estudio presentado es de tipo expost-facto, dado que se requirió información sobre la percepción que tienen los profesores acerca de las prácticas y procedimientos organizacionales; y de las condiciones y el medio ambiente de trabajo de la organización universitaria.

La población conformada fue de 1372 profesores; de los cuales 1281 son ordinarios activos y 91 contratados. A los efectos de analizar las propiedades psicométricas del

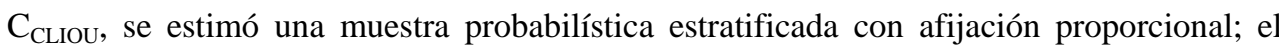
criterio de estratificación empleado fue por Decanatos (Medicina, Ciencia y Tecnología, Agronomía, Ciencia Veterinarias, Ingeniería Civil, Administración y Contaduría) y Departamentos. El cálculo de la muestra se estimó con un nivel de confianza de 95\% y un error de muestreo del 5\%. La muestra fue de 300 profesores, se valoró una mortalidad de 21\%, centrada en el Decanato de Administración y Contaduría, debido a diversas causas, entre las que se mencionan: falta de disposición de los profesores para responder el cuestionario, apatía, desinterés y tiempo. La muestra definitiva la conformaron 271 profesores.

La variable clima organizacional se asumió como un conjunto de percepciones que tienen los profesores sobre las prácticas y procedimientos organizacionales; y de las condiciones y el medio ambiente de trabajo de la organización universitaria. Se midió mediante cuatro (4) componentes y catorce (14) subcomponentes:

1. Imagen Gerencial: Definida como la percepción que tienen los profesores sobre el comportamiento de su jefe inmediato en la institución universitaria, medida mediante el estilo de gerencia, estilo de liderazgo y confianza en la gerencia. Estos subcomponentes fortalecieron el significado conferido a la imagen en la organización universitaria por cuanto se identificaron las cualidades personales del gerente y el estilo de liderazgo o comportamiento observado en la gerencia.

2. Relaciones Sociales: Referida a las percepciones que tienen los profesores sobre la calidad del ambiente social en la organización universitaria, las cuales fueron determinadas a través de la comunicación, cooperación, respeto y relaciones interpersonales. Estos 
subcomponentes consolidaron el significado que se dio al trabajo cooperativo y a las relaciones de equipo en la universidad.

3. Toma de Decisiones: Citada como la percepción que tienen los profesores sobre la actuación de su jefe inmediato en el proceso de la toma de decisiones para escoger la alternativa más ajustada y así lograr un eficiente manejo organizacional. Se valoró mediante la delegación de la toma de decisiones, comunicación y conflictos de intereses. Estos subcomponentes fortalecieron el significado que se concedió a la accesibilidad y viabilización de los procesos gerenciales que son elementos coadyuvantes para un comportamiento organizacional favorable en la organización.

4. Tarea: Expresada como la percepción que tienen los profesores acerca de las condiciones en las cuales realiza el proceso de trabajo, condiciones éstas que definen la calidad y las consecuencias del mismo. Incluyó la orientación en la producción, sobre carga de trabajo, condiciones ergonómicas, y responsabilidad. Estos subcomponentes evalúan la actividad ejecutada por el trabajador y las condiciones de trabajo, es decir, ambiente y recursos existentes para desarrollarla.

Se empleó como instrumento un cuestionario estructurado en dos partes, una referida a datos de identificación (Decanato, departamento y sección) y la otra, a la percepción del clima organizacional que tiene el profesor de su área de trabajo, la cual se desagregó en componentes y subcomponentes. Esta segunda parte se confeccionó en un formato cerrado y sencillo, con una escala de respuesta que emplea los adjetivos: Siempre (5); Casi Siempre (4); A veces (3); Casi Nunca (2); Nunca (1). Ciento dieciocho (118) ítemes o reactivos conformaron la primera versión del instrumento; correspondiendo tres (3) ítemes a cada subcomponente, de acuerdo con los criterios expuestos por Edwars, citado por Ruiz Bolívar (2002) preservando una amplia muestra del contenido al que se refiere el constructo.

Las propiedades psicométricas del $\mathrm{C}_{\mathrm{CLIOU}}$ se determinaron mediante: validez de contenido y validez de constructo; análisis discriminativo y confiabilidad. Para obtener la validez de contenido se emplearon dos procedimientos: el juicio de expertos y el coeficiente de concordancia de Kendall; se seleccionaron 12 profesionales de diferentes universidades del país (UCLA, UPEL-Rubio, UNY), con suficiente conocimiento y experiencia para evaluar el instrumento. La validez de contenido mediante el juicio de expertos se procesó con una muestra de nueve (9) profesionales denominados expertos o jueces, integrados por tres (3) metodólogos, tres (3) profesores versados en el diseño de instrumento y tres (3) con dominio en el tema clima organizacional; estos expertos evaluaron el contenido de los ítemes y su correspondencia con los componentes y subcomponentes que conforman el instrumento.
Para estimar la validez de contenido por medio del coeficiente de concordancia de Kendall, se seleccionaron tres (3) profesionales ubicados en el área de la gerencia, quienes clasificaron cada uno de los ítemes de los catorce (14) subcomponentes definidos para estudiar el clima organizacional en la universidad. Este coeficiente determinó la concordancia que los jueces mostraron al establecer la correspondencia entre ítemes y subcomponentes. Una vez realizadas las observaciones pertinentes se elaboró la segunda versión del cuestionario en igual formato de presentación y escala de respuesta.

Previo al análisis discriminativo de ítemes, se realizó un análisis de correlación de cada ítem con respecto al total general de cada componente, con el finalidad de excluir los ítemes, que obtuviesen una correlación menor o igual a 0,20 (Kerlinger, 2002). El análisis discriminativo de ítemes se realizó con el propósito de conservar solamente aquellos que tuvieran suficiente poder discriminativo; es decir, aquellos ítemes que mostraran un patrón diferencial de respuesta entre dos grupos de sujetos cuyos puntajes globales (total general de ítemes y cada componente) fueran extremos. Este análisis se realizó mediante la prueba “t” de Student para muestras independientes. Después del análisis, se incorporaron mejoras al instrumento y se conformó la tercera versión del cuestionario en igual formato de presentación y escala de respuesta.

La validez de constructo, se hizo mediante el análisis factorial para cada componente estudiado. Se aplicó el método de Componentes Principales con rotación varimax. El análisis de cada componente se hizo tomando en cuenta los pesos factoriales, empleando el criterio de valores iguales o superiores a 0,30. (Visauta, 2000). La confiabilidad se estimó mediante el índice Alpha de Cronbach a todos los ítemes resultantes de la estructura factorial, en general y por cada componente.

\section{PRESENTACIÓN Y DISCUSIÓN DE LOS RESULTADOS}

Las propiedades psicométricas del $\mathrm{C}_{\text {CLIOU }}$ se resumen a través de la validez de contenido, análisis discriminativo de ítemes, validez de contructo y análisis de confiabilidad.

\section{Validez de Contenido}

La validez de contenido del $\mathrm{C}_{\mathrm{CLIOU}}$ se efectuó por una parte, con el fin de determinar la correspondencia entre el contenido de cada ítem y el componente al cual representa. Al respecto, Hernández, Fernández y Baptista (2002) señalan que la evaluación de las preguntas por parte de un grupo de jueces expertos en el área garantiza que el instrumento 
realmente mide la variable que pretende medir. La validez de contenido efectuada por jueces expertos en las áreas de Gerencia, Metodología y Clima Organizacional realizado a ciento dieciocho (118) ítemes del instrumento elaborado en su primera versión produjo los resultados que se describen más adelante.

El análisis crítico de cada ítem determinó que los jueces coincidieran en que fueran eliminados los ítemes 4, 8, 10, 12, 21, 50, 52, 57, 63, 66, 73, 83, 96, 104, 114, 115, 116, 118 por ser imprecisos, contener ideas repetidas, expresiones inadecuadas y presentar sesgo. Para los ítemes 5, 17, 23, 44, 45, 91, 94, 97, 98, 99, 102, 105, 106 13, 18, 27, 30, 34, 40, 53, 60, 67, 78, 84, 85, 90, 92, 98, 109 sugirieron pequeños ajustes, como: colocación de comas, puntos, completación de palabras y cambio de verbos en dos reactivos.

Por otra parte, se propusieron cambios en el nombre de los subcomponentes: centralización por niveles de la toma de decisiones; funciones por desempeño de funciones; sobrecarga de trabajo por asignación de la carga de trabajo; agentes peligrosos por condiciones ambientales, con la finalidad de ofrecer mayor amplitud al área del estudio. Estas recomendaciones no modificaron la definición conceptual del subcomponente. De igual modo, sugirieron agregar en el componente relaciones sociales, el subcomponente respeto y reconocimiento.

Revisadas y analizadas las observaciones, se procedió a hacer los ajustes sugeridos y eliminar los reactivos indicados; así como conservar aquellos ítemes que, según los expertos representaron adecuadamente el dominio. Después de este análisis, el instrumento quedó conformado por cien (100) ítemes distribuidos en cuatro (4) componentes (Imagen Gerencial, Relaciones Sociales, Toma de Decisiones y la Tarea); y catorce subcomponentes distribuidos por ítemes. En general, los jueces expertos concluyeron que los cien (100) ítemes del instrumento presentaron claridad, coherencia y precisión, además opinaron que el formato empleado para su presentación es adecuado.

La validez de contenido se determinó a través del Coeficiente de Concordancia de Kendall, W, utilizado para medir el grado de acuerdo entre jueces respecto a los ítemes de los subcomponentes que conforman el instrumento. Se consideraron los valores entre 0 y 1 (cero indica total desacuerdo entre los jueces; mientras que valores próximos a uno indican total acuerdo), expuestos por Ferrán (2001). Este análisis de concordancia fue realizado por tres (3) profesionales ubicados en el área de la gerencia quienes clasificaron cada uno de los ítemes de los catorce (14) subcomponentes definidos para estudiar el clima organizacional en la universidad. El coeficiente obtenido fue $\mathrm{W}=0,90$. Este valor es indicativo de una consistencia y concordancia entre los jueces expertos para justificar el grado de pertinencia de los ítemes respeto a cada subcomponente estudiado. Dicho valor es superior al reportado para otros instrumentos (Mujica de G y Pérez de M, ob.cit), que han incluido la validez mediante el índice de concordancia entre jueces.

La información anterior se presenta en este estudio como evidencia de validez de contenido en el $\mathrm{C}_{\mathrm{CLIOU}}$ reportada por ambos grupos de expertos, quedando configurada la segunda versión del instrumento por cien (100) ítemes con igual estructura.

\section{Análisis de Ítemes}

El análisis de ítemes se realizó para los componentes: Imagen Gerencial, Relaciones Sociales, Toma de Decisiones y la Tarea, mediante dos procedimientos: el primero un análisis de correlación de ítemes, con el objeto se efectuar una depuración de la base datos, utilizando como referencia los valores de correlación menor o igual a 0,20 (Kerlinger, ob.cit.). En el componente Imagen Gerencial el análisis de los ítemes 16, 18, y 25 exhibió una correlación por debajo de la establecida $(r=0,173 ; 0,055 ; 0,129)$ el resto de ítemes correlacionaron positivamente con una correlación ubicada en la escala que osciló entre alta y muy alta $(r=0,619$ a 0,809$)$. Se constató el grado de consistencia de cada ítem con su respectivo componente. El nivel de significación estadística fue menor al nivel de 0,001 .

Los valores de las correlaciones del componente Relaciones Sociales, reportaron que los ítemes 30, 31, 34, 38, 50, no correlacionaron, ( $\mathrm{r}=-0,128 ; 0,163 ; 0,132 ;-0,022 ; 0,135)$ respectivamente, así como también los ítemes 30 y 38 los cuales mostraron una correlación negativa. Veinticinco ítemes correlacionaron positivamente en una escala que varió entre alta y muy alta $\quad(r=0,609$ y 0,843), excepto el ítem 39, cuya correlación fue moderada ( $r$ $=0,545)$. El nivel de significación estadística fue menor al nivel de 0,001.

Por otra parte, en el componente Toma de Decisiones de un total de veinticuatro (24) ítemes, sólo dos (2) no correlacionaron, correspondiendo a los ítemes 55 y 62 ( $\mathrm{r}=0,191$ y -0,142), este último correlacionó negativamente. El resto de ítemes mostró una correlación positiva, que varió entre moderada, $(\mathrm{r}=0,555)$, a la escala alta y muy alta $(\mathrm{r}=0,641 \mathrm{y}$ 0,847). La significación estadística fue menor al nivel de 0,001. 
Con respecto al componente Tarea; del total de ítemes, sólo doce (12) correlacionaron positivamente y oscilaron valores de correlación entre $r=0,286$ y 0,566, (baja y moderada); el resto mostró ítemes (positivos y negativos) que no correlacionaron por presentar valores por debajo del valor preestablecido $(0,20)$. La significación estadística de los ítemes que correlacionaron fue menor a 0,001 .

Los valores obtenidos en el coeficiente de correlación de Pearson, permitieron estudiar la importancia estadística de la relación entre las variables por medio del análisis de la significación de la asociación encontrada. Los resultados del análisis de correlaciones por cada componente determinó que veintiséis (26) ítemes no correlacionaron, distribuidos de la manera siguiente: Imagen Gerencial (16, 18, y 25), Relaciones Sociales (30, 31, 34, 38, 50) Toma de Decisiones (55 y 62 ) y la Tarea (75, 76, 77, 78, 79, 80, 81, 82, 83, 84, 86, 98, 99, 100), por registrar correlaciones negativas y valores por debajo de lo preestablecido en el estudio (Kerlinger, ob.cit.). Los demás ítemes exhibieron correlaciones positivas. En su mayoría mostraron correlaciones que variaron entre altas y muy altas, siendo el nivel de significación estadística de $\mathrm{p}<0,001$. En conclusión setenta y seis (76) ítemes de los cien (100) correlacionaron positivamente.

Al examinar las intercorrelaciones, se encontró que la Imagen Gerencial mostró una correlación alta, positiva y significativamente con las Relaciones Sociales $(\mathrm{r}=0,866 \mathrm{p}<$ 0,001) y la Toma de Decisiones ( $\mathrm{r}=0,841 \mathrm{p}<0,001)$. Las Relaciones Sociales correlacionó alta, positiva y significativamente con la Toma de Decisiones $\quad(\mathrm{r}=0,846 \mathrm{p}<0,001)$, siendo moderadamente y positiva con la Tarea $(0,537 ; \mathrm{p}<0,05)$. De igual modo, la Toma de Decisiones mostró una correlación moderadamente y positiva con la Tarea $(\mathrm{r}=0,468 \mathrm{p}<$ $0,05)$.

En general los valores de las intercorrelaciones oscilaron entre 0,448 y 0,866 con una valoración que varió entre moderada a muy alta. Además se confirmó que los componentes considerados para medir el clima organizacional en la universidad, correlacionaron entre sí en las combinaciones determinadas.

El segundo análisis de ítemes, fue un análisis discriminativo mediante la prueba t para muestras independientes. El análisis fue realizado a los setenta y seis (76) ítemes del $\mathrm{C}_{\mathrm{CLIOU}}$ que sobrevivieron al análisis de correlación, previo al análisis, se verificaron los supuestos de normalidad y homogeneidad de las varianzas entre grupos (homocedasticidad), ésta última valorada mediante la prueba de Levene cuyo resultado permitió determinar que la probabilidad obtenida, mayor que 0,05 lleva a suponer que las varianzas poblacionales son iguales.

El primer análisis discriminativo se efectuó con los puntajes correspondientes al total general, compuesto por setenta y seis (76) ítemes, con el objeto de mantener solo aquellos que revelaron tener suficiente poder discriminativo; es decir, los ítemes que presentan valor diferencial de respuestas en los dos grupos de sujetos cuyos puntajes totales fuesen extremos. La comparación de cada ítem con el total general, dio como resultado que la totalidad de ítemes mostraron tener suficiente poder discriminativo a un nivel de significación estadística de $\mathrm{p}<$ 0,001; excepto los ítemes 89, 95, 96 que alcanzaron niveles de significación de 0,05.

El segundo análisis se hizo para cada uno de los componentes: Imagen Gerencial, Relaciones Sociales, Toma de Decisiones y la Tarea. Se evidenció que todos los ítemes que agrupan el componente Imagen Gerencial mostraron tener suficiente poder discriminativo; los niveles de significación para la diferencia de medias correspondiente a cada ítem, superaron el nivel preestablecido de 0,05. En suma los veintidós (22) ítemes discriminaron de manera satisfactoria entre los docentes que manifestaron su percepción sobre la Imagen Gerencial del directivo en la universidad.

En el componente Relaciones Sociales los veinte (20) ítemes discriminaron satisfactoriamente a un nivel de significación estadística de $\mathrm{p}<0,0001$. Asimismo el componente Toma de Decisiones mostró que veintidós (22) ítemes analizados reflejaron alto poder discriminativo. En el análisis de ítemes se muestra que de un total de doce (12) ítemes del componente Tarea, sólo diez (10) revelaron tener suficiente poder discriminativo a un nivel de significación estadística de $\mathrm{p}<0,0001$; los restantes se ubicaron con un nivel de $\mathrm{p}<0,05$. El análisis discriminativo realizado a los setenta y seis (76) ítemes evidenció una capacidad discriminativa, tanto al tomar los ítemes en su totalidad, como por componentes.

Los datos reportados para los componentes: Imagen Gerencial, Relaciones Sociales y Toma de Decisiones, mostraron tener suficiente poder discriminativo; los niveles de significación estadística superaron los valores preestablecidos $(\mathrm{p}<0,0001)$. No obstante, el grupo de ítemes en el componente Tarea alcanzó niveles de significación de $\mathrm{p}<0,0001$ y $\mathrm{p}<0,05$. 
En síntesis, el análisis de ítemes mostró, que los setenta y seis ítemes (76) que conforman el instrumento correlacionan positiva y significativamente con la puntuación total, y al tiempo que cada uno de ellos correlacionan positiva y significativamente con la puntuación total correspondiente al componente al que pertenece. Los resultados confirman que los ítemes del instrumento discriminan de manera satisfactoria. La información resultante lleva a esperar que estos ítemes se comporten apropiadamente con la finalidad de identificar la percepción sobre el clima universitario en los docentes.

\section{Validez de Construcción}

El estudio de validez de construcción del instrumento se hizo mediante un análisis factorial con el objetivo de resumir la información obtenida de las variables iniciales que fueron nombradas subcomponentes, expresando las mismas como combinación lineal de otras variables no observables denominadas factores. La validez de construcción de cada componente (Imagen Gerencial, Relaciones Sociales, Toma de Decisiones y la Tarea) se efectuó estudiando en primer lugar, el resultado obtenido de la matriz de componentes principales, posteriormente se aplicó el método de rotación varimax. A partir de este resultado se eliminaron los ítemes según el criterio de saturación expuesto por Visauta (2000) que refiere un modelo en el que todos los ítemes saturen en algún factor, es decir que presenten cargas factoriales altas en uno y bajos en otros; así como pesos factoriales iguales o superiores a 0,30. Una vez tomada esta decisión de eliminar ítemes, se analizaron las cargas factoriales y nuevamente cada componente fue sometido a un proceso de rotación varimax, para obtener una estructura más simple y clara de los factores en cada componente. A continuación se describen los resultados obtenidos.

a) Componente Imagen Gerencial: Veintidós ítemes del componente Imagen Gerencial distribuido en tres subcomponentes: estilo de gerencia, estilo de liderazgo y confianza en la gerencia; fueron sometidos al procedimiento de análisis factorial, previa evaluación de los criterios: índice de adecuación de la muestra de Kaiser- Meyer-Olkin (KMO) y el Test Bartlett, para verificar la procedencia o no del análisis. Los resultados exhibieron un índice KMO de 0,951 el cual mostró un valor excelente, que indica que los datos provienen de una población homogénea y el tamaño de la muestra es adecuado. El Test Bartlett resultó estadísticamente significativo (Aprox. $\chi^{2}=4596,013 \mathrm{p}<0,000$ ), probó que las variables no están correlacionadas en la población; es decir, se comprueba que la matriz de correlaciones no es una matriz de identidad.
El análisis factorial de componentes principales extrajo dos factores que explicaron $62,90 \%$ de la varianza total. El porcentaje de varianza explicada para cada uno de estos factores fue 53,42\% y 9,48\% respectivamente; asimismo las raíces características (o eigenvalues) fueron 11,75; 2,08. Esta selección estuvo basada en el criterio desarrollado por Kaiser citado por Visauta (ob, cit.) el cual consiste en elegir tantos factores como raíces características o valores propios (eigenvalues) sean mayores a la unidad. Estos valores se interpretaron como la cantidad de varianza explicada por cada factor. (Visauta, ob.cit).

Los resultados obtenidos en los pesos factoriales, según el método de componentes principales, varió entre 0,828 y 0,612 en concordancia con lo esperado; el factor I aglutinó la totalidad de subcomponentes: estilo de gerencia, estilo de liderazgo y confianza en la gerencia. No obstante, con el fin de lograr una solución factorial que permita obtener factores independientes se realizó una rotación de los mismos aplicando el método varimax, en el sentido de que las variables fuertemente correlacionadas entre sí presentan saturaciones altas sobre un mismo factor y bajas sobre el resto, en correspondencia con lo expuesto por Visauta (ob.cit.). Es importante destacar que al examinar la matriz de componentes rotados se evidenció que de los veintidós ítemes analizados, después de rotada la matriz, los ítemes 12, 13, 15 y 24, reportaron un nivel de saturación en ambos factores, por lo que se decidió su eliminación, el resto cargó un peso factorial igual o mayor a 0,30 en uno u otro factor.

La solución factorial obtenida quedó estructurada por dos factores representados por dieciocho (18) ítemes, por lo cual se volvió a realizar la rotación. En conclusión, en la tabla 1 se muestra la estructura factorial definitiva que agrupa a los 18 ítemes del componente Imagen Gerencial en dos factores. El primer factor estuvo constituido por ítemes provenientes del subcomponente denominado gerente como orientador; es decir ítemes referidos a las actividades de orientación que realiza el gerente en la organización universitaria. 
Tabla 1

Estructura de la matriz de los 18 Ítemes del Componente Imagen Gerencial en Dos Factores, Segunda Rotación Método Varimax

\begin{tabular}{|c|c|c|c|}
\hline \multirow{2}{*}{$\begin{array}{c}\text { Item } \\
N^{\circ}\end{array}$} & \multirow[t]{2}{*}{ Contenido de los ítemes } & \multicolumn{2}{|c|}{ Cargas Factoriales } \\
\hline & & 1 & 2 \\
\hline 2 & Orienta conflictos & 0,724 & 0,359 \\
\hline 3 & Demuestra interés en el cumplimiento de otros & 0,796 & 0,362 \\
\hline 6 & Canaliza problemas & 0,861 & 0,231 \\
\hline 7 & Apoya el trabajo docente & 0,819 & 0,302 \\
\hline 8 & Facilita el desempeño & 0,811 & 0,302 \\
\hline 9 & Propicia discusión para resolver conflictos & 0,736 & 0,367 \\
\hline 14 & Trabajo fluido & 0,741 & 0,363 \\
\hline 17 & Delega tareas & 0,688 & 0,264 \\
\hline 19 & Brinda apoyo cuando realiza el trabajo docente & 0,642 & 0,465 \\
\hline 22 & Atiende cuando se necesita & 0,837 & $-0,056$ \\
\hline 1 & Supervisa el desempeño docente & $\mathbf{0 , 2 7 3}$ & 0,709 \\
\hline 4 & Orienta funciones docentes & 0,365 & 0,630 \\
\hline 5 & Evalúa el desempeño docente & $\mathbf{0 , 1 3 2}$ & 0,812 \\
\hline 10 & Realiza acciones en función de resultados & 0,192 & 0,750 \\
\hline 11 & Registra resultados de invstigaciones & $\mathbf{0 , 2 1 9}$ & 0,649 \\
\hline 20 & Informa su gestión al finalizar el año & $\mathbf{0 , 2 4 8}$ & 0,686 \\
\hline 21 & Informa los alcances y metas de trabajo & $\mathbf{0 , 3 3 1}$ & 0,735 \\
\hline 23 & Programa discusión del rendimiento estudiantil & $\mathbf{0 , 3 8 1}$ & 0,646 \\
\hline
\end{tabular}

El segundo factor estuvo constituido por ítemes que derivados del subcomponente designado gerente como líder; es decir, incluyó actividades que permiten entender el liderazgo como una visión que asegura el éxito dentro de la organización universitaria. Nótese que los dieciocho (18) ítemes exhiben cargas factoriales de 0,630 ó más en uno de los dos factores, lo que explica una clara definición de cada ítem en un factor.

b) Componente Relaciones Sociales: Veinte ítemes del componente Relaciones Sociales distribuido en cinco (5) subcomponentes: relaciones interpersonales, respeto, reconocimiento, ambiente de cooperación y responsabilidad. Previo al análisis factorial se evaluaron los criterios: índice Kaiser- Meyer-Olkin (KMO) y el Test Bartlett resultante para verificar la procedencia o no del análisis. Los resultados exhibieron un índice KMO de
0,941, el cual mostró un valor excelente e indicó que los datos provienen de una población homogénea y que el tamaño de la muestra es adecuado. El Test Bartlett resultó estadísticamente significativo (Aprox. $\chi^{2}=3795,958 \mathrm{p}<0,000$ ), lo cual indica que las variables no están correlacionadas en la población; es decir, se comprueba que la matriz de correlaciones no es una matriz de identidad.

El análisis factorial de componentes principales extrajo dos factores que explicaron $61,79 \%$ de la varianza total. El porcentaje de varianza explicada para cada uno de estos factores fue $54,47 \%$ y 7,32\% respectivamente. Del mismo modo las raíces características (o eigenvalues) fueron 10,35; 1,39. Esta selección estuvo basada en el criterio desarrollado por Kaiser citado por Visauta (ob, cit.), el cual consiste en elegir tantos factores como raíces características o valores propios (eigenvalues) sean mayores a la unidad. Estos valores se interpretaron como la cantidad de varianza explicada por cada factor. (Visauta, ob.cit.).

Los pesos factoriales, según el método de componentes principales, varió entre 0,851 y 0,496, en concordancia con lo esperado. El factor I aglutinó la totalidad de subcomponentes: relaciones interpersonales, respeto, reconocimiento, ambiente de cooperación y responsabilidad. Con el fin de lograr una solución factorial que permitiera obtener factores independientes se realizó una rotación de los mismos aplicando el método varimax, en el sentido de que las variables fuertemente correlacionadas entre sí presentan saturaciones altas sobre un mismo factor y bajas en las restantes. Al examinar la matriz de componentes rotados, se evidenció que los veinte (20) ítemes equilibraron las cargas de los pesos factoriales por encima de 0,30 en uno u otro factor.

La solución factorial obtenida quedó estructurada por dos factores representados por veinte (20) ítemes, por lo cual se volvió a realizar la rotación. En síntesis, la tabla 2 muestra la solución factorial definitiva que agrupa a los 20 ítemes del componente Relaciones Sociales en dos factores. El primer factor estuvo constituido por ítemes provenientes del subcomponente denominado ambiente de trabajo; es decir ítemes referidos a las actividades de ayuda entre profesores, responsabilidad del trabajo y relaciones interpersonales dentro del ambiente de trabajo.

El segundo factor estuvo constituido por ítemes derivados del subcomponente designado trabajo en conjunto; es decir, incluyó actividades que implican relaciones humanas; así como el enfoque del trabajo como ayuda entre los individuos y los grupos para lograr la efectividad y la productividad del mismo. Nótese que los veinte (20) ítemes 
exhiben cargas factoriales de 0,557 ó más en uno de los dos factores, lo que explica una clara definición de cada ítem en un factor.

\section{Tabla 2}

Estructura de la Matriz de los 20 Ítemes del Componente Relaciones Sociales en Dos Factores, Segunda Rotación Método Varimax

\begin{tabular}{|c|l|c|c|}
\hline Item Contenido de los ítemes & \multicolumn{2}{|c|}{ Cargas Factoriales } \\
\cline { 2 - 3 }$N^{\circ}$ & & \multicolumn{2}{|c|}{$\mathbf{2}$} \\
\hline $\mathbf{2 7}$ & Trato amable hacia los profesores & 0,779 & $\mathbf{0 , 2 5 7}$ \\
\hline $\mathbf{2 8}$ & Ambiente cordial & 0,809 & $\mathbf{0 , 1 2 0}$ \\
\hline $\mathbf{2 9}$ & Actividades consensuadas & 0,768 & $\mathbf{0 , 3 5 3}$ \\
\hline $\mathbf{3 3}$ & Aprecia opiniones de los profesores & 0,672 & $\mathbf{0 , 4 9 0}$ \\
\hline $\mathbf{3 5}$ & Libertad para expresarse & 0,758 & $\mathbf{- 0 , 0 7 8}$ \\
\hline $\mathbf{3 7}$ & Sugerencias para mejorar la planificación & 0,592 & $\mathbf{0 , 2 3 5}$ \\
\hline $\mathbf{4 2}$ & Ayuda entre compañeros & 0,755 & $\mathbf{0 , 2 4 2}$ \\
\hline $\mathbf{4 3}$ & Planifica en grupo & 0,535 & $\mathbf{0 , 3 2 4}$ \\
\hline $\mathbf{4 4}$ & Compromiso compartido & 0,642 & $\mathbf{0 , 2 2 1}$ \\
\hline $\mathbf{4 8}$ & Intercambio de conocimiento & 0,602 & $\mathbf{0 , 3 7 2}$ \\
\hline $\mathbf{2 6}$ & Promueve el trabajo en equipo & $\mathbf{0 , 5 0 7}$ & 0,602 \\
\hline $\mathbf{3 2}$ & Evalúa la programación en equipo & $\mathbf{0 , 2 5 8}$ & 0,729 \\
\hline $\mathbf{3 6}$ & Recibe sugerencias de los estudiantes & $\mathbf{0 , 4 7 1}$ & 0,557 \\
\hline $\mathbf{3 9}$ & Iniciativas de trabajo & $\mathbf{0 , 0 1 5}$ & 0,717 \\
\hline $\mathbf{4 0}$ & Reconocimiento & $\mathbf{0 , 5 4 5}$ & 0,690 \\
\hline $\mathbf{4 1}$ & Promueve las iniciativas & $\mathbf{0 , 4 4 0}$ & 0,671 \\
\hline $\mathbf{4 5}$ & Ejecuta las actividades en equipo & $\mathbf{0 , 4 7 8}$ & 0,626 \\
\hline $\mathbf{4 6}$ & Evalúa la ejecución de actividades en equipo & $\mathbf{0 , 1 5 2}$ & 0,757 \\
\hline $\mathbf{4 7}$ & Acepta cambios en la planificación del semestre & $\mathbf{0 , 4 7 2}$ & 0,694 \\
\hline $\mathbf{4 9}$ & Cumplimiento del horario de trabajo & $\mathbf{0 , 2 0 3}$ & 0,634 \\
\hline
\end{tabular}

c) Componente Toma de Decisiones: Veintidós ítemes conformaron el componente Toma de Decisiones distribuido en tres (3) subcomponentes: niveles de la toma de decisiones, participación de la toma de decisiones y comunicación para la toma de decisiones. Previo al análisis factorial se evaluaron los criterios: índice Kaiser- MeyerOlkin (KMO) y el Test Bartlett resultante para verificar la procedencia o no del análisis. Los resultados exhibieron un índice KMO de 0,952 el cual mostró un valor excelente e indicó que los datos provienen de una población homogénea y el tamaño de la muestra es adecuado. El Test Bartlett resultó estadísticamente significativo (Aprox. $\chi^{2}=4726,439 \mathrm{p}<$ 0,000 ), indica que las variables no están correlacionadas en la población; es decir, se comprueba que la matriz de correlaciones no es una matriz de identidad.

El análisis factorial de componentes principales extrajo dos factores que explicaron $63,07 \%$ de la varianza total. El porcentaje de varianza explicada para cada uno de estos factores fue 53,51\% y 6,89\% respectivamente. Del mismo modo las raíces características (o eigenvalues) fueron 12,36; 1,15. Esta selección estuvo basada en el criterio desarrollado por Kaiser citado por Visauta (ob, cit.) el cual consiste en elegir tantos factores como raíces características o valores propios (eigenvalues) sean mayores a la unidad. Estos valores se interpretaron como la cantidad de varianza explicada por cada factor. (Visauta, ob.cit.).

Los pesos factoriales, según método de componentes principales, varió entre 0,856 y 0,530 en concordancia con lo esperado; el factor I aglutinó la totalidad de subcomponentes: niveles de la toma de decisiones, participación de la toma de decisiones y comunicación para la toma de decisiones. Con el fin de lograr una solución factorial que permitira obtener factores independientes se realizó una rotación de los mismos aplicando el método varimax, en el sentido de que las variables fuertemente correlacionadas entre sí presentan saturaciones altas sobre un mismo factor y bajas en las restantes.

Al examinar la matriz de componentes rotados (se evidenció que de los veintidós ítemes analizados, después de rotada la matriz, los ítemes 51, 52, 53, 56, 57, 58, 59, 72 , reportaron un nivel de saturación en ambos factores, por lo que se decidió su eliminación, el resto cargó un peso factorial igual o mayor a 0,30 en uno u otro factor.

La solución factorial obtenida quedó estructurada por dos factores representados por catorce (14) ítemes, por lo cual se volvió a realizar la rotación. En suma, la tabla 3 muestra la solución factorial definitiva que agrupa a los 14 ítemes del componente Toma de Decisiones en dos factores. El primer factor estuvo constituido por ítemes provenientes de los subcomponentes denominados niveles de la toma de decisión y participación de la toma de decisión; es decir, el gerente o jefe inmediato acciona las decisiones según los hechos, experiencia y nivel dentro de la organización.

El segundo factor estuvo constituido por ítemes derivados del subcomponente designado comunicación de la toma de decisiones dirigido al grupo de profesores en el área de trabajo. Nótese que los catorce (14) ítemes exhiben cargas factoriales de 0,418 ó 
más en uno de los dos factores, lo que explica una clara definición de cada ítem en un factor.

\section{Tabla 3}

\section{Estructura de la matriz de los 14 Ítemes del Componente Toma de Decisiones} en Dos Factores, Segunda Rotación Método Varimax

\begin{tabular}{|c|l|c|c|}
\hline $\begin{array}{c}\text { Item } \\
N^{\circ}\end{array}$ & \multicolumn{1}{|c|}{ Contenido de los ítemes } & \multicolumn{2}{|c|}{ Cargas Factoriales } \\
\hline $\mathbf{5 4}$ & Jerarquiza problemas en conjunto & 0,717 & $\mathbf{0 , 2 3 2}$ \\
\hline $\mathbf{6 0}$ & $\begin{array}{l}\text { Acepta opinión de profesores para la toma de } \\
\text { decisiones }\end{array}$ & 0,780 & $\mathbf{0 , 3 0 5}$ \\
\hline $\mathbf{6 1}$ & Da participación para la toma de decisiones & 0,721 & $\mathbf{0 , 3 4 4}$ \\
\hline $\mathbf{6 3}$ & Opinión de profesores para el plan de acción & 0,786 & $\mathbf{0 , 1 4 4}$ \\
\hline $\mathbf{6 4}$ & Selecciona criterios para la toma de decisiones & 0,748 & $\mathbf{0 , 2 9 2}$ \\
\hline $\mathbf{6 5}$ & Toma de decisiones para abordar problemas & 0,785 & $\mathbf{0 , 3 1 4}$ \\
\hline $\mathbf{6 6}$ & Planifica la toma de decisiones & 0,819 & $\mathbf{0 , 2 7 6}$ \\
\hline $\mathbf{6 7}$ & Conocimientos en la toma de decisiones & $\mathbf{0 , 2 4 4}$ & 0,761 \\
\hline $\mathbf{6 8}$ & Comunica las decisiones por escrito & $\mathbf{0 , 2 0 1}$ & 0,832 \\
\hline $\mathbf{6 9}$ & Toma de decisiones por escrito & $\mathbf{0 , 2 2 1}$ & 0,782 \\
\hline $\mathbf{7 0}$ & Analiza alternativas para la toma de decisiones & $\mathbf{0 , 6 4 9}$ & 0,551 \\
\hline $\mathbf{7 1}$ & Comunica la toma de decisiones & $\mathbf{0 , 5 2 3}$ & 0,651 \\
\hline $\mathbf{7 3}$ & Divulga la toma de decisiones & $\mathbf{0 , 4 9 4}$ & 0,638 \\
\hline $\mathbf{7 4}$ & Evalúa la toma de decisiones en conjunto & 0,418 \\
\hline
\end{tabular}

d) Componente Tarea: Doce ítemes conformaron el componente Tarea distribuido en dos (2) subcomponentes: asignación de la carga de trabajo y condiciones ambientales. Previo al análisis factorial se evaluaron los criterios: índice Kaiser- Meyer-Olkin (KMO) y el Test Bartlett resultante para verificar la procedencia o no del análisis. Los resultados exhibieron un índice KMO de 0,775, el cual mostró un valor aceptable para continuar el análisis factorial. El Test Bartlett resultó estadísticamente significativo (Aprox. $\chi^{2}=$ $894,514 \mathrm{p}<0,000$ ), lo cual indica que las variables no están correlacionadas en la población; es decir, se comprueba que la matriz de correlaciones no es una matriz de identidad.
El análisis factorial de componentes principales extrajo dos factores que explicaron $60,15 \%$ de la varianza total. El porcentaje de varianza explicada para cada uno de estos factores fue $54,44 \%$ y 5,71\% respectivamente. Del mismo modo las raíces características (o eigenvalues) fueron 4,66; 1,04. Esta selección estuvo basada en el criterio desarrollado por Kaiser citado por Visauta (ob, cit.) el cual consiste en elegir tantos factores como raíces características o valores propios (eigenvalues) sean mayores a la unidad. Estos valores se interpretaron como la cantidad de varianza explicada por cada factor. (Visauta, ob.cit.).

Los resultados obtenidos de los pesos factoriales, según el método de componentes principales, se observó que el factor I aglutinó la totalidad de subcomponentes: asignación de la carga de trabajo y condiciones ambientales; el peso factorial varió entre 0,739 y 0,430 .

Con el fin de lograr una solución factorial que permita obtener factores independientes se realizó una rotación de los mismos aplicando el método varimax, en el sentido de que las variables fuertemente correlacionadas entre sí presentan saturaciones altas sobre un mismo factor y bajas en las restantes. Por otra parte, al examinar la matriz de componentes rotados, se evidenció que los doce (12) ítemes equilibraron las cargas de los pesos factoriales por encima de 0,30 en uno u otro factor.

Al describir la matriz se distingue que el primer factor está compuesto por subcomponentes que agrupan variables que tienen relación con la Tarea identificada con una actividad individual ejecutada por el docente que ocupa un cargo, que a su vez corresponde con la distribución de la carga docente y las asignaciones de trabajo de acuerdo a la dedicación y competencia del trabajador. Este factor se denominó asignación de la carga de trabajo.

El segundo factor está centrado fundamentalmente en el subcomponente que aglutinan variables relacionadas con las condiciones del medio ambiente de trabajo; esto significa que el profesor necesita encontrar un ambiente grato, armónico y confortable donde pernotar para cumplir una función determinada, así como disponer de recursos para ejecutar el trabajo docente - administrativo.

El factor estudiado podría denominarse condiciones ambientales. Ambos factores (asignación de la carga de trabajo y condiciones ambientales) miden como el clima organizacional es expresado por la Tarea que los profesores tienen bajo su responsabilidad. 
La solución factorial obtenida quedó estructurada por dos factores representados por doce (12) ítemes que miden la carga de trabajo del profesor y las condiciones del medio ambiente para llevar a cabo la tarea. Nótese que los doce (12) ítemes exhiben cargas factoriales de 0,420 ó más en uno de los dos factores, lo que explica una clara definición de cada ítem en un factor. Los resultados obtenidos después de la rotación coinciden con los esperados. El primer factor estuvo constituido por ítemes provenientes del subcomponentes denominado asignación de la carga de trabajo, y el segundo factor estuvo integrado por ítemes derivados del subcomponente designado, condiciones ambientales.

\section{Tabla 4}

\section{Estructura de la Matriz de los 12 Ítemes del Componente Tarea en Dos Factores, según Método Rotado Varimax}

\begin{tabular}{|c|l|c|c|}
\hline Item & \multicolumn{1}{|c|}{ Contenido de los ítemes } & \multicolumn{2}{c|}{ Cargas Factoriales } \\
\cline { 3 - 4 }$N^{\circ}$ & & $\mathbf{2}$ \\
\hline $\mathbf{8 5}$ & $\begin{array}{l}\text { Establece diferencias para la distribución del } \\
\text { trabajo }\end{array}$ & $\mathbf{- 0 , 1 8 3}$ & 0,481 \\
\hline $\mathbf{8 7}$ & Ejecuta trabajo solo de competencia & $\mathbf{- 0 , 0 2 2}$ & 0,493 \\
\hline $\mathbf{8 8}$ & Realiza una distribución justa de las actividades & $\mathbf{- 0 , 0 7 7}$ & 0,755 \\
\hline $\mathbf{8 9}$ & Número de profesores suficientes & $\mathbf{0 , 2 6 7}$ & 0,471 \\
\hline $\mathbf{9 0}$ & Opinión del profesor según carga docente & $\mathbf{- 0 , 0 9 3}$ & 0,780 \\
\hline $\mathbf{9 1}$ & Carga docente por dedicación académica & $\mathbf{0 , 1 0 2}$ & 0,805 \\
\hline $\mathbf{9 2}$ & Ambiente de trabajo iluminado & 0,535 & $\mathbf{0 , 2 9 3}$ \\
\hline $\mathbf{9 3}$ & Lugar de trabajo ventilado & 0,696 & $\mathbf{- 0 , 3 4 0}$ \\
\hline $\mathbf{9 4}$ & Disponibilidad de recursos para realizar el trabajo & 0,792 & $\mathbf{0 , 1 1 8}$ \\
\hline $\mathbf{9 5}$ & La silla ofrece una postura ergonómica & 0,727 & $\mathbf{- 0 , 0 1 6}$ \\
\hline $\mathbf{9 6}$ & Postura para ejecutar el trabajo & 0,420 & $\mathbf{- 0 , 1 2 3}$ \\
\hline $\mathbf{9 7}$ & Ambiente agradable & 0,738 & $\mathbf{- 0 , 0 9 9}$ \\
\hline & & & \\
\hline
\end{tabular}

El proceso de análisis psicométrico seguido para el estudio del $\mathrm{C}_{\mathrm{CLIOU}}$ se corresponde con lo planteado por Coyle y otros (ob. cit.); Toro (2001); Mujica de G y Pérez de M (ob. cit.), al utilizar entre otros procedimientos estadísticos el análisis factorial para obtener evidencias de la validez de construcción del $\mathrm{C}_{\mathrm{CLIOU}}$ al lograr una estructura factorial clara y sólida para estudiar el clima organizacional en la universidad. La interpretación de los componentes resultantes en función de los pesos factoriales obtenidos, permitió concluir que emergió una estructura que reveló el pensar de los profesores universitarios; es decir, indicó la manera como éstos sienten o perciben el clima organizacional en su área de trabajo.

En consecuencia, los resultados obtenidos en cada componente después de la rotación del método varimax, permitió un reacomodo en la definición de los catorce (14) subcomponentes planteados al inicio del estudio; quedando un instrumento constituido por ocho (8) subcomponentes conformados por sesenta y cuatro (64) reactivos para medir el clima de trabajo a través de los componentes: Imagen Gerencial, Relaciones Sociales, Toma de Decisiones y la Tarea, de manera relevante y pertinente.

\section{Análisis de Confiabilidad}

La confiabilidad es la exactitud o precisión de un instrumento de medición (Kerlinger, ob.cit). Existen distintos tipos de confiabilidad, entre los que se menciona la congruencia interna, que determina el grado en el cual los ítemes de una prueba están correlacionados entre sí. Se puede poner a prueba mediante diversos métodos. En este estudio el análisis de confiabilidad del instrumento se realizó mediante la prueba estadística Alpha de Cronbach.

Este coeficiente permitió obtener una medida del grado de homogeneidad de los ítemes; es decir, su consistencia interna y el nivel de interrelación de cada ítem (Nunnally y Bernstein 1995., Kerlinger ob.cit.). A tal efecto, se aplicaron los procedimientos de desviación por ítem, totalidad de los ítemes en la escala y para cada grupo de ítem por componentes, con la finalidad de detectar hasta qué punto el citado instrumento permite evaluar o diagnosticar una determinada realidad fiable.

Los resultados de confiabilidad por ítem, incluyendo sólo los sesenta y cuatro (64) ítemes conformaron la versión definitiva del instrumento. Los valores resultantes dan una idea de cuán fiable es la escala utilizada. Los puntajes oscilaron entre 0,973 a 0,975, lo cual expresa que existe una alta correlación entre los ítemes de la escala que revelan su homogeneidad y consistencia interna. Los valores de consistencia interna obtenidos para el instrumento en general y para cada subcomponente se sitúan por encima de 0,80, según 
Ruiz Bolívar (ob.cit.), “un coeficiente de confiabilidad es aceptable cuando está por lo menos en el límite superior $(0,80)$ de la categoría alta” (p.55).

El índice de confiabilidad general del instrumento es de 0,974, esto revela un coeficiente muy alto, valores similares fueron obtenidos para los componentes Imagen Gerencial; Relaciones Sociales y Toma de Decisiones, quienes reportaron índices de confiabilidad de 0,947; 0,949 y 0,944 respectivamente. Dichos coeficientes se ubicaron en la categoría del indicador como muy alto. No obstante, el componente Tarea mostró un coeficiente de 0,879 valorado en la escala como bueno; la magnitud de estos coeficientes establece que cada uno de los componentes y subcomponentes presentan consistencia interna en la medida.

\section{COMENTARIOS FINALES}

En el presente artículo se describen las cualidades psicométricas del Cuestionario Clima Organizacional Universitario ( $\left.\mathrm{C}_{\mathrm{CLIOU}}\right)$ evidenciadas a través de los procedimientos estadísticos empleados para el estudio de validez de contenido y construcción; así como la medida de la confiabilidad.

La validez de contenido se realizó mediante el juicio de expertos en el cual se concluyó que los cien (100) ítemes del instrumento presentaron claridad, coherencia y precisión, de 118 reactivos revisados. La evaluación de las preguntas por parte de un grupo de jueces expertos garantizó que el instrumento realmente mida la variable clima organizacional en el ambiente universitario. Asimismo, hubo coincidencias entre los expertos al manifestar, que el formato empleado es el adecuado. Esta información fue verificada mediante el Índice de Concordancia de Kendall cuyo resultado fue alto $(\mathrm{W}=0,90)$ lo que significa que existe consistencia y concordancia entre los jueces; considerándose lo expuesto coherente con lo señalado por Brown y Holmes (ob.cit.) y Hernández, Fernández y Baptista (ob.cit.), quienes destacan la importancia de evaluar la variable a fin de determinar si mide lo que pretende medir.

La validez de construcción se hizo mediante un análisis factorial con el objetivo de resumir la información obtenida de las variables iniciales que fueron nombradas subcomponentes, expresando las mismas como combinación lineal de otras variables no observables, denominadas factores. Los resultados obtenidos en cada componente después de la rotación del método varimax, permitió un reacomodo en la definición de los catorce
(14) subcomponentes planteados al inicio del estudio; quedando el instrumento constituido por ocho (8) subcomponentes y cuatro (4) componentes: Imagen Gerencial, Relaciones Sociales, Toma de Decisiones y la Tarea. Cada uno definió una estructura bifactorial proveniente de los ítemes de cada subcomponente. Esto significa que el clima organizacional, es una variable multidimensional de naturaleza compleja que se expresa a través de los componentes estudiados. En definitiva, se determinó una estructura factorial sólida acorde con los componentes y subcomponentes propuestos en el instrumento para estudiar el clima organizacional en la universidad. La interpretación de los componentes resultantes en función de los pesos factoriales obtenidos, llevó a concluir que emergió una estructura que reveló el pensar de los profesores universitarios; es decir indicó la manera cómo éstos sienten o perciben el clima organizacional en su área de trabajo.

El Instrumento tipo cuestionario presenta su confiabilidad expresada mediante el Índice Alpha de Cronbach. El Índice total del cuestionario fue de 0,974, lo cual revela un coeficiente muy alto, valores similares resultaron para los componentes Imagen Gerencial, Relaciones Sociales, Toma de decisiones los cuales reportaron índices de confiabilidad de 0,947; 0,949; 0,944 respectivamente. Sin embargo, la Tarea registró un coeficiente catalogado como bueno $(0,879)$. Estos resultados expresan que el instrumento es homogéneo y presenta consistencia interna para medir el clima organizacional en la universidad, puesto que las evidencias presentadas expresan su estabilidad, precisión, seguridad y homogeneidad para la medida de cada componente y subcomponente.

En síntesis, el Cuestionario sobre clima organizacional universitario $\left(\mathrm{C}_{\mathrm{CLIOU}}\right)$ es un instrumento seguro y estable en la medición del contructo que se pretende medir, cuyo diseño original estuvo integrado por 118 ítemes quedando en definitiva conformado por 64 ítemes, cuatro (4) componentes Imagen Gerencial, Relaciones Sociales, Toma de decisiones y la Tarea y ocho (8) subcomponentes: gerente como orientador, gerente como líder; ambiente de trabajo, trabajo en conjunto; participación de la toma de decisiones, comunicación de la toma de decisiones; asignación de la carga de trabajo y condiciones ambientales. Además posee cualidades psicométricas apropiadas para, obtener una medida que informe las condiciones del ambiente universitario según la percepción de los profesores.

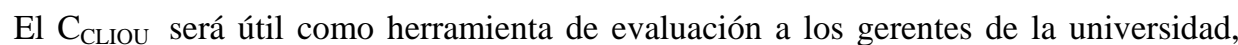
especialmente a los jefes de departamentos y jefes de sección a quienes les corresponde tomar decisiones para mejorar el ambiente de trabajo dirigidas a la implantación de correctivos a corto y mediano plazo. Con la medición del clima, el gerente recibe 
información para mejorar su gestión y con ello podrá promover un ambiente de trabajo que coadyuve en los miembros de la comunidad universitaria, la participación y el desempeño de alta calidad.

\section{REFERENCIAS}

Alvarez, G. (1992). El Constructo “Clima Organizacional”: Concepto, Teorías, Acciones Investigaciones y Resultados Relevantes. Interamericana de Psicología Ocupacional 11, (1,-2).

Brown, R.L. y Holmes, H. (1986). The use of a factoranalytic procedure for assesing the validity of an employee safety climate model. Accident Analysis and Prevention. 18(6): pp. 455-470.

Brunet, L (1999). El Clima de Trabajo en las Organizaciones: definición, diagnóstico y consecuencias. Editorial Trillas. México.

Chiavenato, I. (2001). Introducción a la Teoría General de la Administración. 5ta Edición Editorial. Mac Graw Hill. México D.F.

Coyle, I.R.; Sleeman, S.D. y Adams, N. (1995). Safety Climate. Journal of Safety Research, Vol. 26, nº4, pp.247-254.

Ferrán, M (2001). SPSS para Windows. Análisis Estadístico. McGraw-Hill. Interamericana de España.

García, S (2002). La Validez y la Confiabilidad en la Evaluación del Aprendizaje desde la Perspectiva Hermenéutica. Revista de Pedagogía. 23 (67), 297-318. Caracas.

GonÇalves, A (2000). Clima Organizacional. /página web en línea/. Disponible: http: Clima.Org/ vismeyos.htm/educarchile/ Consulta: 2004 mayo 07.
González, J. M. (2000). Clima Educativo Universitario. Ediciones de la Universidad Ezequiel Zamora. Colección Docencia Universitaria.

Hall, R. (1996). Organizaciones, Estructura, Procesos y Resultados. 2da Edición. Madrid: Editorial Printice Hall Internacional.

Hernández, R., Fernández, C., Baptista, P. (2002). Metodología de la Investigación. México DF. Mc Graw-Hill.

Kerlinger, F y Howard B (2002). Investigación del Comportamiento: métodos de investigación en ciencias sociales. Editorial: McGraw-Hill. 4ta Edición.

Martín Bris, M (2000). Clima de Trabajo y Organizaciones que Aprenden. Educar, 27(2), 103-107.

Mejias, A y otros (2006). Medición del Clñima Organizacional en Instituciones de Educación Superior.Universidad, Ciencia y Tecnología. 10, (38), 55-104.

Meliá, J e Islas, M (1990). Batería de cuestionarios de Seguridad Laboral V3 (ISBN: 84-604-0825-6).

Melia, J y Sesé, A (1999). La medida del clima de seguridad y salud laboral. Anales de psicología. Vol . 15, $\mathrm{N}^{\circ}$ 2, 269-289. Universitat de Valencia. España.

Mujica de González M y Pérez de Maldonado I (2006). Clima Organizacional: Un Indicador de Gestión Universitaria. Tesis Doctoral no publicada. UPELUCLA- UNEXPO. Barquisimeto. Venezuela.

Nunnally, J.C. y Bernstein, I.J. (1995). Teoría Psicométrica. México, D.F.: McGraw-Hill Interamericana.

Ruiz Bolívar, C (2002) Instrumento de Iinvestigación Educativa Procedimientos para su Diseño y Validación. 2da Edición Ediciones EIDEG. Barquisimeto, Venezuela. Pp: 102-104. 
Silva, C (2005). Diseño de un Instrumento para Caracterizar el Clima Organizacional en Unidades Académicas de la Universidad de Colima. Tesis para Optar al Grado de Maestra en Pedagogía no publicada. Universidad de Colima.

Toro, F (2000). Clima Organizacional, Satisfacción del Personal y Percepción de la Calidad de Servicio por los Clientes. Interamericana de Psicología Ocupacional. 19 (1).

Toro, F. (1992). Clima Organizacional y Expectativas en la Perspectiva del Cambio Organizacional. Revista Interamericana de Psicología Ocupacional $11,(1-2)$.

Toro, F. (1998). Predicción del Comportamiento del Personal a parte del Análisis del Clima Organizacional. Interamericana de Psicología Ocupacional. 14 (3).

Toro, F. (2001). El Clima Organizacional: Perfil de las Empresas Colombianas.CINCEL. Medellín-Colombia.

Visauta, V (1999). Análisis Estadístico con SPSS para Window. Vol II. España: Mc-Graw Hill Interamericana.

Visauta, V (2000). Análisis Estadístico con SPSS para Windows. Estadística Básica. Madrid. Editorial McGraw-Hill. 